\title{
Geriatric nutritional risk index serves as risk factor of surgical site infection after pancreatoduodenectomy: a validation cohort Ageo study
}

\author{
Naotake Funamizu ${ }^{1,2}$, Kenji Omura ${ }^{1}$, Takahiro Ozaki ${ }^{1}$, Masayuki Honda ${ }^{1}$, Kohei Mishima ${ }^{1}$, Kazuharu \\ Igarashi $^{1}$, Yasutsugu Takada ${ }^{2}$, Go Wakabayashi ${ }^{1}$ \\ Department of Surgery, Ageo Central General Hospital, Saitama Prefecture, Japan; ${ }^{2}$ Department of HBP Surgery, Ehime University, Ehime \\ Prefecture, Japan \\ Contributions: (I) Conception and design: N Funamizu; (II) Administrative support: K Omura, G Wakabayashi; (III) Provision of study material or \\ patients: None; (IV) Collection and assembly of data: N Funamizu, M Honda, K Mishima; (V) Data analysis and interpretation: N Funamizu, K \\ Igarashi, Y Takada, G Wakabayashi; (VI) Manuscript writing: All authors; (VII) Final approval of manuscript: All authors. \\ Correspondence to: Naotake Funamizu. Department of Surgery, Ageo Central General Hospital, 1-10-10 Kashiwaza, Ageo-City, Saitama Prefecture \\ 362-8588, Japan. Email: funamizujikei@yahoo.co.jp.
}

Background: Surgical site infections (SSIs), which are associated with preoperative malnutrition,
are a well-known potential complication of surgery that leads to increased medical costs and longer
hospitalizations. Thus, surgeons need to accurately identify patients at high-risk for SSIs. Considering that
the Geriatric Nutritional Risk Index (GNRI) was designed to assess the degree of malnutrition specifically
among elderly patients, previous evidence (Kawaguchi study) proved that GNRI predicted the risk of SSIs in
patients following pancreatoduodenectomy (PD). In this study, we aimed to validate whether that the same
index could predict the risk of SSI among patients who underwent PD in our patient cohort (Ageo study).
Methods: The current validation cohort study was retrospectively conducted on 93 patients at the
Department of Surgery, Ageo Central General Hospital, Japan, from January 2015 to October 2019. All
patients were subjected to nutritional screening using the GNRI and were followed up for the occurrence
of postoperative complications. Additionally, risk factors for developing SSI, as well as patient's height, body
mass index, and preoperative laboratory values, were recorded.

Results: Patients were divided into the SSI ( $=30)$ and non-SSI (N=63) groups with a determined SSI incidence rate of $32.3 \%$ (30/93). The SSI group had significantly lower GNRI than the non-SSI group $(\mathrm{P}<0.001)$. Receiver operating characteristic curve analysis determined a cutoff GNRI value of 94 (sensitivity, $83.3 \%$; specificity, $83.6 \%$ ), similar to that in the previous study. Univariate and multivariate analyses confirmed that a GNRI of $<94$ was significantly associated with SSI $(\mathrm{P}<0.001)$.

Conclusions: The present Ageo study confirmed the consistency of results in Kawaguchi study. Thus, lower GNRI can be a universal marker for SSI risk following PD.

Keywords: Pancreatoduodenectomy (PD); geriatric nutritional risk index (GNRI); surgical site infection (SSI); validation study

Submitted Apr 09, 2020. Accepted for publication Nov 12, 2020.

doi: $10.21037 /$ gs-20-451

View this article at: http://dx.doi.org/10.21037/gs-20-451

\section{Introduction}

Pancreaticoduodenectomy (PD) has been the gold standard surgical procedure for malignant hepatobiliary pancreatic and periampullary tumors. With advances in the development of surgical techniques, devices, and perioperative managements, surgical-related mortality 
rates following $\mathrm{PD}$ has decrease to less than 5\% (1-3). However, some studies have still reported morbidity rates of $30-65 \%(4,5)$. Among the potential complications, the most common have generally been surgical site infections (SSIs), delayed gastric emptying and postoperative pancreatic fistula (POPF). In particular, SSIs, including intraabdominal abscess and wound infection, are occasionally caused by POPF (6). SSIs have been known to increase medical costs and prolong hospitalization. Thus, to prevent such outcomes, prompt identification and prevention of SSIs are clinically imperative. More recently, the Geriatric Nutritional Risk Index (GNRI) has gained favor in assessing a patient's nutritional status and predicting clinical outcomes among elderly patients, particularly those with chronic kidney disease and heart failure $(7,8)$. More importantly, the GNRI is easily accessible and inexpensive, requiring only data on body weight, height, and serum albumin levels (9). On the other hand, body weight and albumin levels are associated with postoperative complications including SSI. That was why we hypothesized that GNRI could evaluate the risk of SSI for the patients previously. Due to the intimate relationship between preoperative nutritional status and SSI, previous study (Kawaguchi study) had proved that GNRI $<94$ could predict SSI among patients who underwent PD (10). Thus, the present Ageo study aimed to validate the association between the GNRI and SSI among the patents who underwent PD in our cohort (Ageo study). This is important considering that the identification of predictive markers for SSI may help identify high-risk patients. We herein present the following article in accordance with the STROBE reporting checklist (available at http://dx.doi.org/10.21037/gs-20-451).

\section{Methods}

\section{Patients}

From January 2015 to October 2019, 93 consecutive patients who underwent PD for malignant hepatobiliary pancreatic or duodenal tumors were enrolled. Patients who underwent robotic and laparoscopic PD were excluded. Our protocol had been reviewed and approved by the Ethics Committee of Ageo Central General Hospital in 2019 (approval number: AMG736), and in accordance with the Helsinki Declaration as revised in 2013. All participants, including retrospectively registered patients or their guardians, verbally consented to the use of their medical information for scientific research. Given that the previous study (Kawaguchi study) enrolled 106 patients who underwent PD at Kawaguchi Municipal Medical Center, a sample size of 86 to 126 patients was planned (Ageo study).

\section{Clinical and laboratory data collection}

Clinical data, including demographic variables (gender and age), anthropometric parameters [height, weight, and body mass index (BMI)], comorbidities, smoking history and alcohol intake, American Society of Anesthesiologist (ASA) physical status classification, estimated blood loss, operative time, and laboratory data (albumin, which was chosen from most recent preoperative data), were collected from individual medical records and analyzed until discharge. Anthropometric parameters were rounded off to one decimal place.

\section{Definition of the GNRI}

The GNRI was calculated as follows using serum albumin, height, and body weight obtained on admission: GNRI = $[14.89 \times$ serum albumin $(g / L)]+[41.7 \times$ present/ideal body weight $(\mathrm{kg})]$. Ideal body weight was defined as $22 \times$ patient's height $(\mathrm{m}) \times$ patient's height $(\mathrm{m})$. If the present body weight exceeded the ideal body weight, the present/ideal body weight was set to 1 . After definition of the GNRI cut-off value, the patients were divided into two groups according to the presence of SSIs.

\section{Definition of the SSIs and POPF}

SSIs were diagnosed according to the CDC guidelines. In addition, the definition of PPH and POPF were defined and clarified according to the International Study Group for Pancreatic Surgery (ISGPS) in 2016.

\section{Statistical analysis}

All statistical analyzes were performed using GraphPad Prism v5.0 (GraphPad Software Inc., La Jolla, CA, USA) and SPSS (SPSS Inc., Chicago, IL, USA). Statistical significance was determined using Fisher's exact test or the Chi-squared test. The optimal cutoff value for GNRI was determined using receiver operating characteristic (ROC) curve analysis. Potential risk factors for SSI were determined using univariate (the Chi-squared test or Fisher's exact) and multivariate analyzes. Independent risk factors for SSI were identified using logistic regression. Odds ratios with $95 \%$ confidence intervals were also 
Table 1 Results of univariate analysis

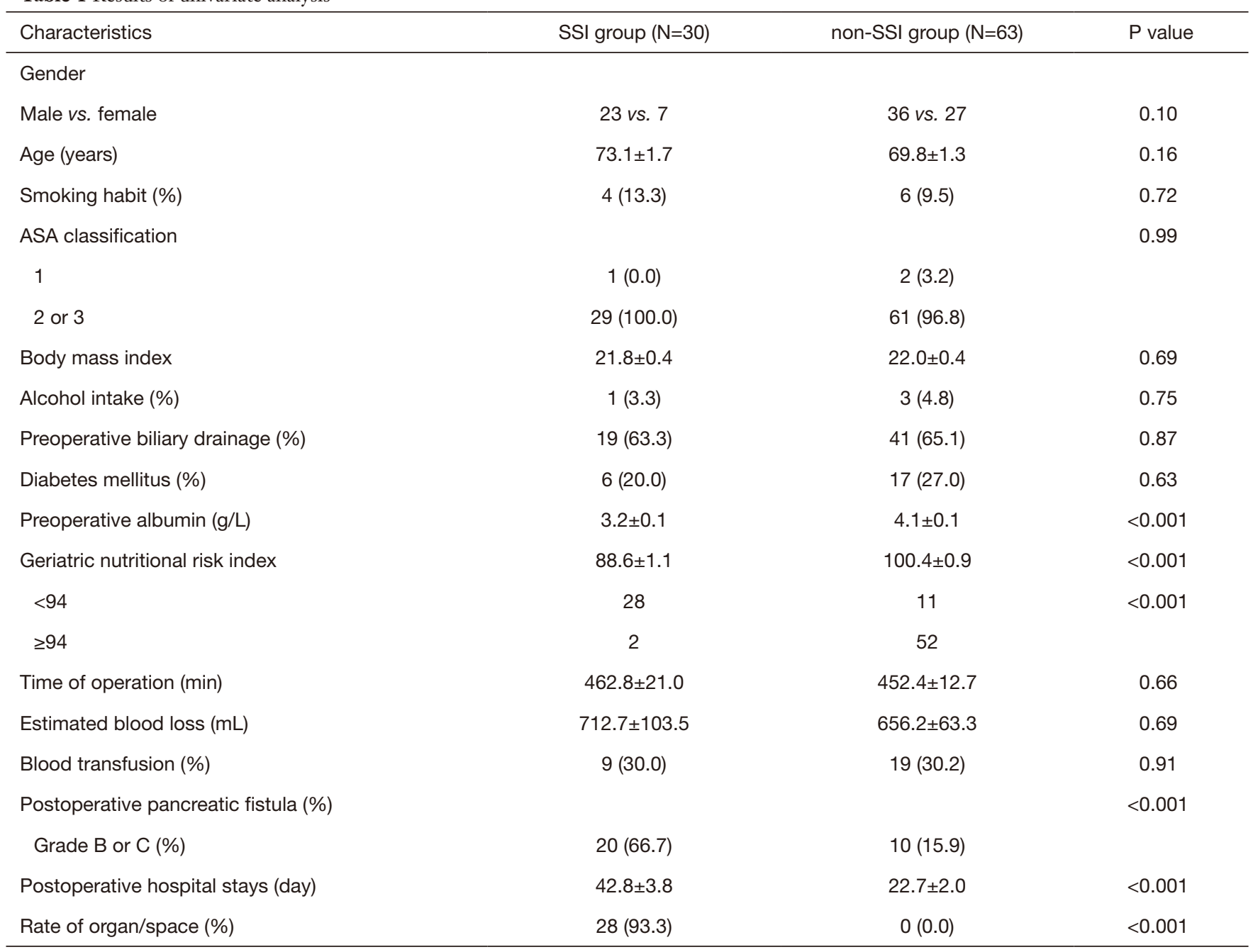

The analysis revealed significantly higher incidence of SSI in GNRI <94 patients.

calculated. A P value of $<0.05$ was considered statistically significant. In this study, the difference of measurement data including albumin is considered as a possible bias factor.

\section{Results}

\section{Patient characteristics}

Patients included herein had a male to female ratio of 1.7:1 $(59 / 34)$ and a mean age of $70.9 \pm 10.1$ years. Among them, 30 patients developed SSIs (32.3\%).

\section{Univariate analysis for SSI risk following PD}

Patients were divided into two groups according to the presence or absence of SSIs. Clinical and demographic data from each group are summarized in Table 1. Although no statistically significant differences in gender, age, smoking habit, ASA classification, BMI, alcohol intake, preoperative biliary drainage, estimated blood loss, operative time, blood transfusions, and postoperative length of hospitalization were observed between the SSI and non-SSI groups, statistically significant differences were observed for preoperative albumin $(\mathrm{P}<0.001)$, GNRI values $(\mathrm{P}<0.001)$, pancreatic fistula $(\mathrm{P}<0.001)$ and postoperative hospital stays $(\mathrm{P}<0.001)$. POPF developed in 30 patients $(32.3 \%), 20$ of whom $(66.7 \%)$ developed SSIs. The latest International Study Group of Pancreatic Surgery criteria reported in 2016 (11) defined POPF as grades B and C complications. Moreover, majority of the SSIs were developed at the 


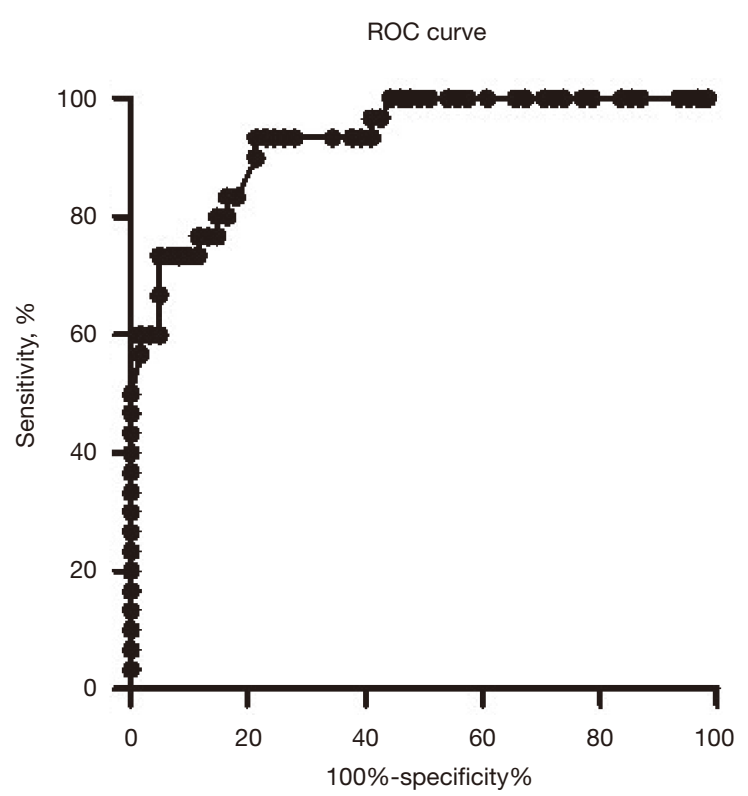

Figure 1 ROC curve analysis. GNRI was chosen by 94 as an optimal cutoff value with sensitivity $83.3 \%$ and specificity $83.6 \%$.

organ/space $(\mathrm{N}=28)$.

\section{Optimal GNRI cutoff value calculation}

The optimal cutoff value was determined using ROC curve analysis (Figure 1). With an area under the curve of 0.93 (95\% confidence interval: $0.88-0.98$ ), the most appropriate cutoff value determined to be 94 , which was the same as that in our previous work. This value had a sensitivity of $83.3 \%$ and a specificity of $83.6 \%$. Patients were then divided into two groups according to the established cutoff value: group A (GNRI $\geq 94, \mathrm{~N}=52$ ) and group B (GNRI $<94, \mathrm{~N}=41$ ). Accordingly, the observed SSI rate in groups A and $\mathrm{B}$ was $3.8 \%$ and $68.3 \%$, respectively.

\section{Univariate analysis for SSI risk following PD}

Univariate analysis revealed that a GNRI value $<94$ was able to predict SSIs risk following PD. Group B had a significantly higher incidence of SSI than group $\mathrm{A}(\mathrm{P}<0.001)$.

\section{Multivariate logistic regression analysis}

Logistic regression analysis showed that a GNRI value $<94$ was an independent risk factor for SSIs as outlined in Table 2.

\section{Discussion}

$\mathrm{PD}$ is a technically complex and invasive procedure. As such, preventing postoperative complications, including POPF, delayed gastric empty, intra-abdominal bleeding, and SSIs, remain a major priority for surgeons. In particular, SSIs, which are among the most common complications following PD, can lead to decreased survival and delayed adjuvant chemotherapy $(6,11)$. In addition, SSIs prolong hospitalization and increase medical costs by an estimated $\$ 1,400$ per patient after PD (12). Additionally, POPF can promote SSIs apart from merely occurring independently. Accordingly, studies have reported that $45-55 \%$ of patients with POPFs developed intra-abdominal abscesses $(13,14)$. Recent reports have also revealed an association between nutritional status and POPF among patients who had undergone pancreatic resection $(15,16)$.

Identified risk factors for SSIs following PD generally include preoperative bile drainage, BMI, age, neoadjuvant chemotherapy, operative time, estimated blood loss, pancreatic duct diameter, and nutritional status (17-19). Despite the absence of definitive risk factors for SSIs, but nutritional status must reach a certain level of consensus among risk factors for developing SSIs in abdominal surgery (20). Another study showed that lower albumin level was an independent predictor of SSIs among patients with gastrointestinal cancer (20). Moreover, other studies have shown that sarcopenia was an objective and independent preoperative predictor of infectious complications after PD (21), while preoperative sarcopenia affected liver surgery outcomes (22) even during liver transplantation (23).

Several objective parameters have been developed to assess nutritional status, such as Nutrition Risk Index, Prognostic Nutritional Index (24), Controlling Nutritional Status (25), and the GNRI (26). Among them, the GNRI had been previously identified as a risk factor for SSIs among patients after PD in Kawaguchi study (10). The GNRI had originally been developed to predict prognosis among elderly patients (9). Subsequently, several studies have consistently reported a relationship between the GNRI and postoperative complications. For instance, one study showed that the GNRI criteria may help in the risk stratification of elderly patients undergoing chemoradiation therapy (27), while, Hanada et al. showed that the GNRI can predict postoperative complications after abdominal surgery and delay postoperative course (28). Moreover, the GNRI can be useful in predicting postoperative 
Table 2 Multivariate analysis by logistical regression

\begin{tabular}{lccc}
\hline Characteristics & Odds ratio & 95\% confidence interval & P value \\
\hline Diabetes mellitus & 0.92 & $0.18-6.78$ & 0.92 \\
Preoperative albumin $(\mathrm{g} / \mathrm{L})$ & 123.79 & $4.41-3,472.55$ & 0.005 \\
Postoperative pancreatic fistula & 9.63 & $1.45-63.95$ & 0.019 \\
Geriatric nutritional risk index $<94$ & 0.113 & $0.01-0.9$ & 0.04 \\
Blood transfusion & 0.2 & $0.02-1.8$ & 0.15 \\
\hline
\end{tabular}

GNRI <94 was independent risk factor to predict SSI following PD.

complications among elderly patients with gastric cancer undergoing gastrectomy (29).

The present Ageo study aimed to validate whether the GNRI can predict the incidence of SSI following PD in our patient cohort. Accordingly, results for the validation cohort revealed that a low GNRI value $(<94)$, which was the same as that in previous Kawaguchi study (10), was strongly associated with higher SSI risk, supporting the feasibility of nutritional assessment before surgery. Recent evidence by Sasaki et $a l$. also showed that the GNRI could be a useful predictor of SSI after surgery, further supporting our data (30). Thus, we believe that our hypothesis has exhibited relative consistency. The present Ageo study has several limitations worth noting. First, though our hypothesis had been confirmed in two independent cohorts, the relatively small sample size may limit the statistical power of our study. Second, given the retrospective design of this study, a more comprehensive prospective study should be conducted to confirm our findings in the near future.

\section{Conclusions}

The present study provided assurance of Kawaguchi study that a lower GNRI, which can be easily calculated using serum albumin level, height, and body weight, can be a potential predictor of SSIs among patients who had undergone $\mathrm{PD}$ in clinical practice.

\section{Acknowledgments}

The authors thank Dr. Noriko Funamizu (Department of Internal Medicine, Hirose Hospital, Ehime, Japan) for their advice and discussion.

Funding: None.

\section{Footnote}

Reporting Checklist: The authors have completed the STROBE reporting checklist. Available at http://dx.doi. org/10.21037/gs-20-451

Data Sharing Statement: Available at http://dx.doi. org/10.21037/gs-20-451

Conflicts of Interest: All authors have completed the ICMJE uniform disclosure form (available at http://dx.doi. org/10.21037/gs-20-451). The authors have no conflicts of interest to declare.

Ethical Statement: The authors are accountable for all aspects of the work in ensuring that questions related to the accuracy or integrity of any part of the work are appropriately investigated and resolved. The present study was approved by the Ethics Committee of Ageo Central General Hospital in 2019 (approval number: AMG736) and informed verbal consent obtained from each patient. In addition, approval of the present study was performed according to the Declaration of Helsinki as revised in 2013.

Open Access Statement: This is an Open Access article distributed in accordance with the Creative Commons Attribution-NonCommercial-NoDerivs 4.0 International License (CC BY-NC-ND 4.0), which permits the noncommercial replication and distribution of the article with the strict proviso that no changes or edits are made and the original work is properly cited (including links to both the formal publication through the relevant DOI and the license). See: https://creativecommons.org/licenses/by-nc-nd/4.0/. 


\section{References}

1. Satoi S, Takai S, Matsui Y, et al. Less morbidity after pancreaticoduodenectomy of patients with pancreatic cancer. Pancreas 2006;33:45-52.

2. Rosenberg L, MacNeil P, Turcotte L. Economic evaluation of the use of octreotide for prevention of complications following pancreatic resection. J Gastrointest Surg 1999;3:225-32.

3. Kimura W, Miyata H, Gotoh M, et al. A pancreaticoduodenectomy risk model derived from 8575 cases from a national single-race population (Japanese) using a web-based data entry system: the 30-day and inhospital mortality rates for pancreaticoduodenectomy. Ann Surg 2014;259:773-80.

4. Ahmad SA, Edwards MJ, Sutton JM. Factors influencing readmission after pancreaticoduodenectomy: a multi-institutional study of 1302 patients. Ann Surg 2012;256:529-37.

5. Büchler MW, Wagner M, Schmied BM, et al. Changes in morbidity after pancreatic resection: toward the end of completion pancreatectomy. Arch Surg 2003;138:1310-4.

6. DeOliveira ML, Winter JM, Schafer M. Assessment of complications after pancreatic surgery: a novel grading system applied to 633 patients undergoing pancreaticoduodenectomy. Ann Surg 2006;244:931-7.

7. Komatsu M, Okazaki M, Tsuchiya K, et al. Geriatric Nutritional Risk Index Is a Simple Predictor of Mortality in Chronic Hemodialysis Patients. Blood Purif 2015;39:281-7.

8. Kaneko H, Suzuki S, Goto M, et al. Geriatric nutritional risk index in hospitalized heart failure patients. Int J Cardiol 2015;181:213-5.

9. Funamizu N, Nakabayashi Y, Iida T, et al. Geriatric nutritional risk index predicts surgical site infection after pancreaticoduodenectomy. Mol Clin Oncol 2018;9:274-8.

10. Bassi C, Marchegiani G, Dervenis C, et al. The 2016 update of the International Study Group (ISGPS) definition and grading of postoperative pancreatic fistula: 11 years after. Surgery 2017;161:584-91.

11. Dimick JB, Chen SL, Taheri PA, et al. Hospital costs associated with surgical complications: a report from the private-sector National Surgical Quality Improvement Program. J Am Coll Surg 2004;199:531-7.

12. Parikh JA, Beane JD, Kilbane EM, et al. Is American College of Surgeons NSQIP organ space infection a surrogate for pancreatic fistula? J Am Coll Surg 2014;219:1111-6.

13. Kimura F, Shimizu H, Yoshidome H, et al. Increased plasma levels of IL-6 and IL-8 are associated with surgical site infection after pancreaticoduodenectomy. Pancreas 2006; 32:178-85.

14. Sato N, Tamura T, Minagawa N, et al. Preoperative body mass index-to-prognostic nutritional index ratio predicts pancreatic fistula after pancreaticoduodenectomy. Hepatobiliary Surg Nutr 2016;5:256-62.

15. Funamizu N, Nakabayashi Y, Kurihara K. Lower geriatric nutritional risk index predicts postoperative pancreatic fistula in patients with distal pancreatectomy. Mol Clin Oncol 2020;12:134-7.

16. Watanabe M, Miyata H, Gotoh M, et al. Total gastrectomy risk model: data from 20,011 Japanese patients in a nationwide internet-based database. Ann Surg 2014;260:1034-9.

17. Sugiura T, Uesaka K, Ohmagari N, et al. Risk factor of surgical site infection after pancreaticoduodenectomy. World J Surg 2012;36:2888-94.

18. Poruk KE, Lin JA, Cooper MA, et al. A novel validated risk score to predict surgical site infection after pancreaticoduodenectomy. HPB (Oxford) 2016;18:893-9.

19. Kuzu MA, Terzioğlu H, Genç V, et al. Preoperative nutritional risk assessment in predicting postoperative outcome in patients undergoing major surgery. World J Surg 2006;30:378-90.

20. Bozzetti F, Gianotti L, Braga M, et al. Postoperative complications in gastrointestinal cancer patients: the joint role of the nutritional status and the nutritional support. Clin Nutr 2007;26:698-709.

21. Takagi K, Yoshida R, Yagi T, et al. Radiographic sarcopenia predicts postoperative infectious complications in patients undergoing pancreaticoduodenectomy. BMC Surg 2017;17:64.

22. Kaido T, Hamaguchi Y, Uemoto S. Significance of preoperative sarcopenia to liver surgery. Hepatobiliary Surg Nutr 2019;8:59-62.

23. Pagano D, Barbieri L, Seidita A, et al. Importance of sarcopenia parameter changes after living donor liver transplantation. Hepatobiliary Surg Nutr 2017;6:193-5.

24. Alvares-da-Silva MR, Reverbel da Silveira T. Comparison between handgrip strength, subjective global assessment, and prognostic nutritional index in assessing malnutrition and predicting clinical outcome in cirrhotic outpatients. Nutrition 2005;21:113-7.

25. Ignacio de Ulíbarri J, González-Madroño A, de Villar NG, et al. CONUT: a tool for controlling nutritional status. First validation in a hospital population. Nutr Hosp 2005;20:38-45. 
26. Bouillanne O, Morineau G, Dupont C, et al. Geriatric Nutritional Risk Index: a new index for evaluating at-risk elderly medical patients. Am J Clin Nutr 2005;82:777-83.

27. Wang Y, Wang L, Fang M, et al. Prognostic Value of the Geriatric Nutritional Risk Index in Patients Exceeding 70 Years Old with Esophageal Squamous Cell Carcinoma. Nutr Cancer 2020;72:620-6.

28. Hanada M, Yamauchi K, Miyazaki S, et al. Geriatric Nutritional Risk Index, a predictive assessment tool, for postoperative complications after abdominal surgery: A

Cite this article as: Funamizu N, Omura K, Ozaki T, Honda M, Mishima K, Igarashi K, Takada Y, Wakabayashi G. Geriatric nutritional risk index serves as risk factor of surgical site infection after pancreatoduodenectomy: a validation cohort Ageo study. Gland Surg 2020;9(6):1982-1988. doi: 10.21037/gs20-451 prospective multicenter cohort study. Geriatr Gerontol Int 2019;19:924-9.

29. Kushiyama S, Sakurai K, Kubo N, et al. The Preoperative Geriatric Nutritional Risk Index Predicts Postoperative Complications in Elderly Patients with Gastric Cancer Undergoing Gastrectomy. In Vivo 2018;32:1667-72.

30. Sasaki H, Nagano S, Taniguchi N, et al. Risk Factors for Surgical Site Infection after Soft-Tissue Sarcoma Resection, Including the Preoperative Geriatric Nutritional Risk Index. Nutrients 2018 ;10:1900. 